\title{
D CC CRYOSTAT TEST COOLDOWN - COOLDOWN TIME
}

\author{
J.D. FUERST
}

AUGUST 19, 1987

D) NOTE \#3740.000-EN107 


\section{Summary:}

The Dq CC Cryostat is to be cold tested with $\mathrm{LN}_{2}$.

Calculations ahow that the time required for the 12.5 ton atainlese eteel inner vesael to reach equilibrium is around 5 houra if the ressel is cooled by introducing liquid into a norsle at the bottom. The heat transer calculations contain many aseumptions, however. As a reault, the veasel will be cooled by eprajing $\mathrm{LN}_{2}$ through a nozzle at the vessel top, providing as fast a cooldown as desired.

\section{Discussion:}

The cooldown was modeled as if the veseel at room temperature ouddenly contained $3000 \mathrm{gal}$. (50") of $\mathrm{LN}_{2}$ pooled in the bottom. As the calculatione that follow show, conduction proved to be an insignificant mode of heat tranefer (hundreds of ratts). Inaccuracies include the asoumption of one dimensional heat flow through a uniform crose section.

Radiation was treated with the asoumption of a constant average temperature difference between ressel wall and cold sink, giving a heat trangfer rate of thousands of watts.

Convection was modeled after a vertical plate geometry under free convection and turbulent flow. Given these asaumptione and an arerage (conetant) temperature difference, the conrection heat tranefer coefficient was calculated to be $12 \mathrm{~W} / \mathrm{m}^{2} \mathrm{~K}$. This indicated a beat tranafer rate of about 150,000 watta - by far the dominant mode of heat tranefer.

With these rates and the known amount of heat in the vessel (mass times enthalpy change from $300 \mathrm{~K}$ to $80 \mathrm{~K}$ ), the cooldown time is readily calculated.

The end of this note contains more detailed calculations by T.J. Peteraon considering conrection only (a reasonable assumption) and taking into account the decaying nature of the temperature difference between ressel wall and gas. This calculation provides the 5 hour cooldown estimate. 


\section{Conclusion:}

Although calculations of the bottom-fill cooldown method indicate a reasonable cooldown time, the asaumption of uniform gas temperature (absence of stratification) is vital to the analysis and in fact may not bo valid.

Initially, as liquid is introduced into the bottom of the ressol, it will boil rapidly creating large amounts of cold gas which then cool the walls above. As the ressel bottom cools and $\mathrm{LN}_{2}$ begins to pool, however, the boiloff rate could decrease aignificantly. Thus the cold gas assumed in the free convection calculations is not generated. For this reason and in the interest of a speedy cooldown it has been decided to fill the vessel by spraying $\mathrm{LN}_{2}$ in through a nozale in the vessel top. 
COOLPOWN TIME

- consider condinitions connection, radiation.

Conduction:

$$
\dot{Q}=K \frac{\mathrm{A}}{\mathrm{L}}
$$

where $\begin{aligned} R= & \text { thermal conductivity integral for atm. st l. from } \\ & 300 \text { to } 100 \mathrm{~K}=2532 \mathrm{w/m}\end{aligned}$

$A=$ cross -sectional heat traverser area. Although this value changes as the "coff wave "progres sos is the vessel walls, a value determined at the vessel "equator" will be used (cirnumorimex X wall thickness) $=[2(488 \mathrm{~cm})+2(305 \mathrm{~cm})](1.59 \mathrm{~cm})=2522 \mathrm{~cm}^{2}$

$L=$ lengthof conduction path up side $\approx 500 \mathrm{~cm}$

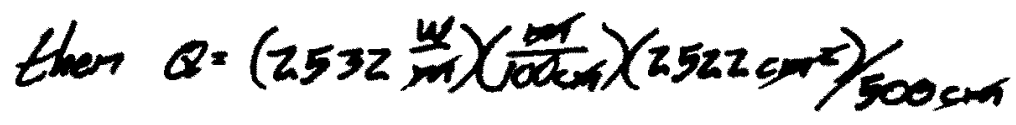
$=128 \mathrm{~W}$

Convection :

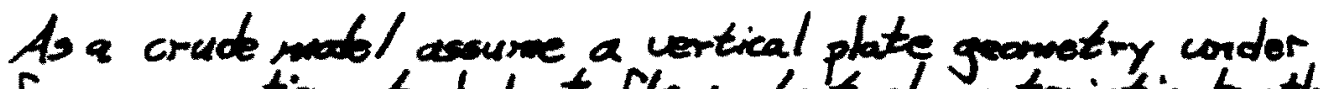
free correction, turbulent flow. Let characteristic berg th of grancetry "' he equal to the length of arc from liquid edge to top of Vessel. (50 atm)

I st assume $\Delta T$ from 300 to $100 \mathrm{~K}$, then 300 to $200 \mathrm{~K}$

so $\bar{h}=\frac{\overline{\mu_{u} K}}{L}$ and $\overline{N_{u}}-\left\{0.825+\frac{0.387 R_{2}^{1 / 6}}{\left[1+\left(0.492 / p_{r}\right)^{9 / 10}\right]^{0 / 2}}\right\}^{2}$

where $h_{a}=$ Raykigh number $=G_{r} P_{r}=\frac{g B\left(T_{0}-T_{L}\right) L^{3}}{\nu \alpha}$

$B=$ volumetric therm. exp. coif. $\approx \frac{1}{T_{L}}=0.01 \mathrm{~K}^{-1}$

$$
\begin{array}{ll}
g=9.81 \mathrm{~m} / \mathrm{s} & D=2 \times 10^{-6} \mathrm{~m}^{2} / \mathrm{s} \\
T_{0}=300 \mathrm{~K} & \alpha=2.6 \times 10^{-6} \mathrm{~m}^{2} / \mathrm{s} \\
T_{L}=100 \mathrm{~K} & R=0.768 \\
L=5 \mathrm{~m} & K=9.58 \times 10^{-3} \mathrm{w} / \mathrm{mK}
\end{array}
$$




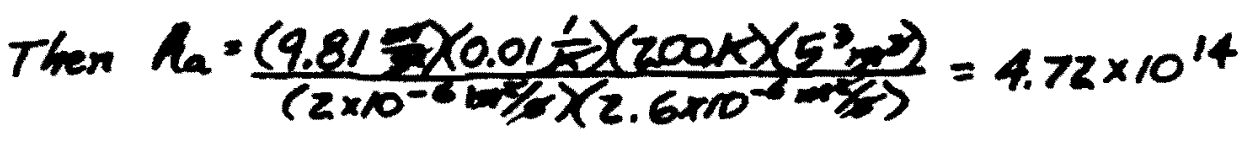
and $\overline{\mu_{s}}=\left[0.825+\frac{107.97}{1.186}\right]^{2}=8439$ finally $\bar{h}=\frac{(8439) \times 0.00958 \mathrm{w} / \mathrm{mk})}{5 \mathrm{~m}}=16.17 \mathrm{w} / \mathrm{m}^{2 \mathrm{~K}}$

if $\Delta T$ from 300 to 200 , then

$$
\begin{aligned}
& v=7.65 \times 10^{-6} \mathrm{~m} 2 / \mathrm{s} \\
& a=10.4 \times 10^{-6} \mathrm{~m} / \mathrm{s} \\
& P_{n}=0.736 \\
& k=18.3 \times 10^{-3} w_{1 k} \\
& B=0005 \mathrm{k}^{-1} \\
& R_{a}=7.71 \times 10^{12}, N_{u}=2166, \bar{h}=7.93 \mathrm{w} / \mathrm{m}^{2 \mathrm{~K}}
\end{aligned}
$$

I will assume a comet. any value of $12 \mathrm{~W} /$ ark for simplicity.

Tat transirate $Q=h A \Delta T$ where

$$
\begin{aligned}
& h=12 \mathrm{w} / \mathrm{m} 2 \mathrm{k} \\
& \Delta T=150 \mathrm{~K} \text { (and.) } \\
& A=\text { inside sur face area of warm pertof } \\
& \text { vessel = cylinder area }+ \text { end undlarea } \\
& =\pi d L+2 \pi r^{2} \\
& \left.=\pi[488 \mathrm{~cm})(305 \mathrm{~cm})+2(244 \mathrm{~cm})^{2}\right] \\
& =840000 \mathrm{~cm}^{2}=84 \mathrm{~m}^{2} \\
& Q=(12 \mathrm{w}=18 \mathrm{w})(8 \mathrm{~m})(150 \mathrm{k})=151200 \mathrm{~W}
\end{aligned}
$$

Radiation:

$$
\begin{aligned}
& Q=\in \sigma A\left(T_{0}^{4}-T_{4}^{4}\right) \\
& \epsilon=\text { minisisity }=0.22 \\
& \sigma=\text { Stefor-atteman cost. }=5.67 \times 10^{-8} \mathrm{w} / \mathrm{m} / \mathrm{k} \\
& T_{\infty}=300 \mathrm{~K} \\
& T_{L}: 150 \mathrm{~K} \text { (aug.) } \\
& A=\text { same as for convection }=84 \mathrm{~m}^{2} \\
& \text { so } Q=(.22)\left(5.67 \times 10^{-84} / 5 \times 1\right)\left(7.591 \times 10^{4} \times 1\right)(84,000) \\
& =7960 \mathrm{~W}
\end{aligned}
$$









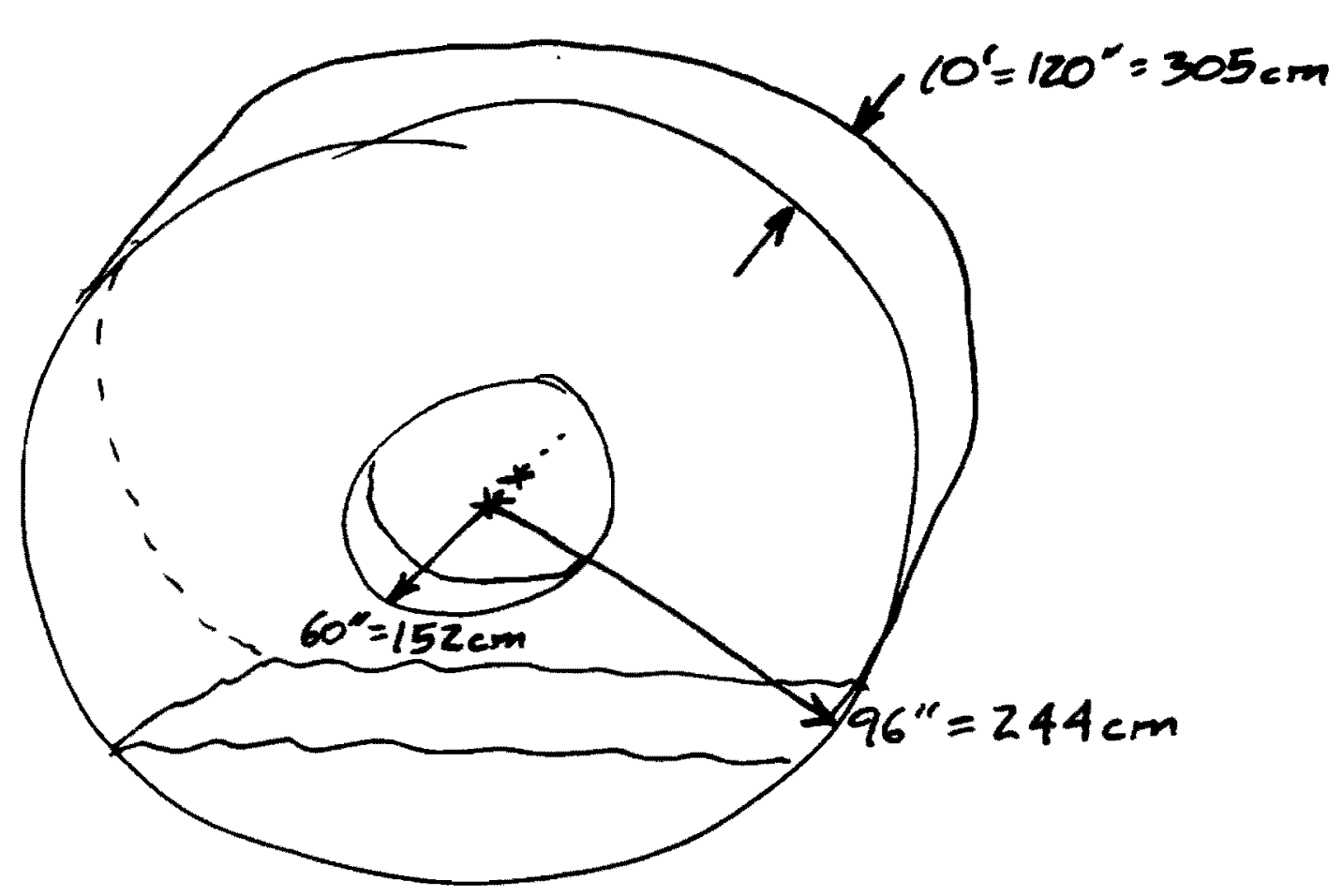

Surface area inside (neglect liquid pool):(\$ hole)

$$
\begin{aligned}
& A_{c y l}+A_{\text {end }}+A_{\text {and }} \\
& 2 \pi r L+2 \pi r^{2} \\
& 2 \pi\left[(244 \mathrm{~cm})(305 \mathrm{~cm})+\left(244 \mathrm{~cm}^{2}\right]=840000 \mathrm{~cm}^{2}=84 \mathrm{~m}^{2}\right.
\end{aligned}
$$

assume effects of liquid pool (remove surf. area) and hole (add curntiarea) more or 
Jo -l,

1 Seen mo.

$$
\begin{aligned}
& \dot{E}_{\text {out }}=\dot{E}_{\text {staved }} \\
& \text { Ton } \\
& \dot{E}_{\text {out }} \cong h A\left(T_{\text {wall }}-T_{\text {go: }}\right) \\
& \dot{E}_{\text {toed }}=m c \frac{d T_{w}}{d t} \text {. } \\
& \text { y } \frac{1}{3} \frac{k}{3} \\
& -h \bar{A}\left(T_{w}-T_{g}\right)=m c \frac{d T_{w}}{d t} \\
& =m c \frac{d\left(T_{w}-T_{g}\right)}{d t} \\
& -\frac{h A}{m c} d t=\frac{d\left(T_{w}-I_{g}\right)}{T_{w}-T_{g}} \\
& -\frac{h A t}{m c}+\text { conan }=\ln \left(T_{w}-T_{g}\right) \\
& \operatorname{const}_{2} e^{-\frac{h A}{m c} t}=T_{w}-T_{g} \\
& t=0 \Rightarrow \quad T_{w}-T_{y}=\quad T_{w i}-T_{g} \\
& \therefore \text { cont }_{2}=T_{w i}-T_{g}
\end{aligned}
$$$$
\left(T_{w_{i}}-T_{g}\right) e^{\frac{h A}{m c} t}=T_{w}-T_{g}
$$ 
2

dont meed a. Tg

$$
\begin{aligned}
& h=12 \mathrm{w} / \mathrm{m}^{2} \mathrm{~K} \\
& A=84 \mathrm{~m}^{2} \text {. } \\
& m c=\left(12.5^{\operatorname{tana}}\right)\left(\frac{2000 \mathrm{~b}^{\prime}}{\mathrm{m}}\right)\left(\frac{\mathrm{H1} .514 \mathrm{~kg}}{32.74 \mathrm{lb}}\right)\left(\frac{500 \mathrm{~J}}{\mathrm{~kg} \mathrm{~K}}\right. \\
& =5.67 \times 10^{6} \mathrm{~J} / \mathrm{K} \\
& \frac{h A}{m c}=\frac{12 \times 84}{5.67 \times 10^{6}}-s^{-1}=1.78 \times 10^{-4} s^{-1}
\end{aligned}
$$

$$
\frac{T_{w}-T_{y}}{T_{w i}-T_{y}}=e^{\left(-1.78 \times 10^{-4}\right) t}
$$

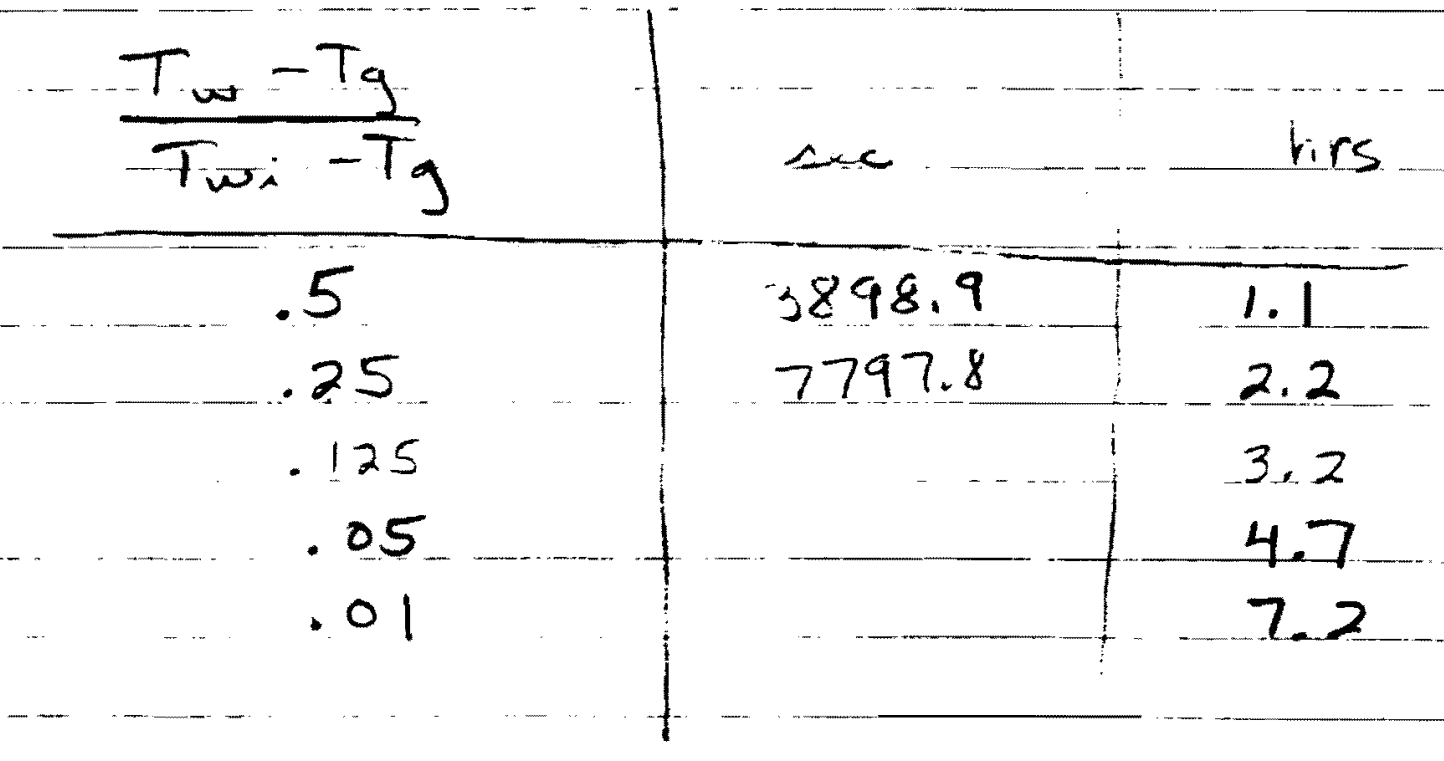

$$
t=-5.62 \times 10^{3} \ln \left(\frac{I_{w}-T_{g}}{T_{w i}-T_{g}}\right)
$$


3

Vessel will operate at $25: K$ $\mathrm{LN}_{2}$ is at about $78 \mathrm{~K} / \mathrm{T}=98$

$$
\frac{T_{w}-T_{g}}{T_{w i}-T_{g}}=\frac{88-78}{300-78}=.05
$$

Takes 5 hrs

Thermal contraction of $55 x$ is $91 \%$ complete at $90 \mathrm{~K}$ 